\title{
Double Blinded, Randomized and Controlled Comparative Study Evaluating the Cleaning Activity of Two Ear Cleaners in Client-Owned Dogs with Spontaneous Otitis Externa
}

\author{
Geneviève Marignac ${ }^{1}$, Jean Yanique Petit ${ }^{2}$, Jean François Jamet ${ }^{3}$, Loic Desquilbet ${ }^{2}$, \\ Jean Luc Petit ${ }^{4}$, Frédérique Woehrlé5, Tessa Trouchonn ${ }^{5}$, Oscar Fantini ${ }^{*}$, Sébastien Perrot ${ }^{2}$ \\ ${ }^{1}$ Dermatology Derpartment, Ecole Nationale Vétérinaire d'Alfort, Maisons-Alfort, France \\ ${ }^{2}$ Institut of Alfort's Clinical Research, IRCA, Ecole Nationale Vétérinaire d'Alfort, Maisons-Alfort, France \\ ${ }^{3}$ Veterinary Clinic, Choisy le Roi, France \\ ${ }^{4}$ Global Medical, Marketing \& Communication, Vetoquinol SA, Magny-Vernois, Lure, France \\ ${ }^{5}$ Preclinical \& Clinical Development, Global Drug Development Division, Vétoquinol SA, Magny-Vernois, Lure, France \\ Email: *oscar.fantini@vetoquinol.com
}

How to cite this paper: Marignac, G., Petit, J.Y., Jamet, J.F., Desquilbet, L., Petit, J.L., Woehrlé, F., Trouchon, T., Fantini, O. and Perrot, S. (2019) Double Blinded, Randomized and Controlled Comparative Study Evaluating the Cleaning Activity of Two Ear Cleaners in Client-Owned Dogs with Spontaneous Otitis Externa. Open Journal of Veterinary Medicine, 9, 67-78. https://doi.org/10.4236/ojvm.2019.96006

Received: May 20, 2019

Accepted: June 27, 2019

Published: June 30, 2019

Copyright ( 2019 by author(s) and Scientific Research Publishing Inc. This work is licensed under the Creative Commons Attribution International License (CC BY 4.0).

http://creativecommons.org/licenses/by/4.0/

(c) $\underset{\mathrm{EY}}{\text { (i) Open Access }}$

\begin{abstract}
Ear cleaning is a therapeutic component in the management of otitis externa in dogs. The objectives of this study were to evaluate the in vivo efficacy and safety of a new ear cleanser, Sonotix ${ }^{\bullet}$ against EpiOtic ${ }^{\bullet}$ Advanced for the management of canine otitis externa. Eighteen clients owned dogs with a diagnosis of erythemato-ceruminous or purulent otitis externa were prospectively included and randomized to two treatment group: EpiOtic ${ }^{\varpi}$ Advanced and Sonotix ${ }^{\oplus}$. Cytology and video-otoscopic examination (erythema, amount of cerumen and thickness and surface of ear canal covered by cerumen) of all affected ears were done at $\mathrm{D} 0$, both before (T0) and 30 minutes (T0 + $30 \mathrm{~min}$ ) after ear cleaning. Then an ear medication was applied (Aurizon`, Vetoquinol). Owners were instructed to clean affected ears daily and apply the ear medication 30 minutes later for 5 days (D1-D5). Dogs were seen again at D6 for cytology and video-otoscopic examination. At T0, no significant differences were found between both ear cleansers groups regarding macroscopic and microscopic scorings. At T0 $+30 \mathrm{~min}$ and D6 cytological and video-otoscopic scores were significantly decreased (Wilcoxon test; $\mathrm{p}<0.01$ ) compared to baseline in both groups. However, the cleaning activity of Sonotix ${ }^{\otimes}$ was statistically superior to Epiotic ${ }^{\circledR}$ as evidenced by the median global scores of video-otoscopic examination at $\mathrm{T} 0+30 \mathrm{~min}$ (Mann Whitney Test, $\mathrm{p}$ $<0.01)$. Effective ear cleaning is an essential part of any treatment scheme because it favours the contact between the ointment and the lining ear epi-
\end{abstract}


dermis and speeds resolution by the removal of cerumen, microbial organisms and cellular debris. In our study, the important reductions in yeast, cocci, and rod-shaped organism counts were demonstrated in smears at T0 + 30 min and D6 in both groups. Video-otoscopic examination performed 30 minutes after ear cleaning suggests that Sonotix ${ }^{\otimes}$ seems to be more effective in removing cerumen than EpiOtic ${ }^{\circledast}$ Advanced.

\section{Keywords}

Ear Cleaning, Canine Ear Cleanser, Otitis Externa

\section{Introduction}

Otitis externa (OE) is a common pruritic and/or painful condition characterized by skin inflammation of the ear canal that can be related to a complex and multifactorial aetiology [1] [2] [3]. Causes of otitis are various, involving primary factors such as hypersensitivities and ectoparasites, predisposing factors are related to ear anatomy and environmental conditions, secondary factors that exacerbate inflammation such as bacterial/fungal infections and perpetuating factors that prevent the resolution of otitis and lead to relapse [1] [3]. Reporting rate from veterinary clinics indicated that canine $\mathrm{OE}$ is a very common condition (4\% - 20\%) [4] [5].

Chronic OE is associated with a thickening of the ear canal walls, stenosis, malfunctioning of secretory system and colonization by pathogenic microorganisms [2] [6].

Aggregated cerumen, debris and exudates are factors favouring the overgrowth of pathogens such as Staphylococcus pseudintermedius, Pseudomonas aeruginosa and Malassezia pachydermatis [7] [8] [9] [10]. Therefore, the diagnosis $\mathrm{OE}$ relies on a clinical history as well as a clinical examination of the ear canal and cytology of the ear exudates [1] [11] [12].

Ear cleaning is part of routine canine hygiene for many owners [4] [13]. It is frequently used prior to otic ointment application and to maintain a favourable ear environment, especially for dogs with predisposing factors. Treatment of $\mathrm{OE}$ is usually local and ear cleansers are often prescribed as the first-line treatment for OE before any medicated topical treatment is applied. The aim of the cleansing agents is to clean the surface of the ear canal lining epidermis, to remove microbes, biofilms, debris and small foreign bodies, to expose the lining of the ear canal to topical therapy and to prevent inactivation of topical therapy [13]. A wide range of ear cleansers with different properties are available on the veterinary market [13] [14] [15] [16] but given the high degree of complexity in the formulation and the limited particularly on its detailed composition, it is difficult for the practitioner to have reliable data to evaluate their properties. In literature, very few in vivo or in vitro studies of ceruminolytic activity of veterinary ear cleaning products have been conducted [17] [18] [19] [20]. In a previous in 
vitro trial whose aim was to compare the ceruminolytic activity of five commercial ear cleaners on synthetic canine cerumen, the ear solution (Sonotix ${ }^{\oplus}, \mathrm{Ve}$ toquinol, Lure, France) containing ethoxydyglycol, isopropyl alcohol, capric glycerides, lipacids, glycerin, thromethamine, polysorbate 80 and calendula had the most effective and rapid ceruminolytic activity of the products tested [17].

The purpose of this study is to investigate and to compare the safety and the efficacy of a new commercial ear cleaner (Sonotix ${ }^{\oplus}$, Vetoquinol, Lure, France) in comparison to a commercial canine ear cleaner (EpiOtic ${ }^{\circledast}$ Advanced, Virbac, Carros France) to reduce cerumen accumulation and micro-organisms in the external ear canal of client-owned dogs with spontaneous otitis externa.

\section{Materials \& Methods}

\subsection{Overview}

Dogs with a visible amount of cerumen and spontaneous otitis externa were prospectively included between December, 14th, 2014 and June, 12th, 2015. The study was conducted in compliance with applicable animal welfare regulations relating to the care and use of animals for scientific purposes, and the study protocol was approved by the relevant institutional Animal Ethics. The study was conducted in accordance with good clinical practice guidelines (GCP) and written informed consent was obtained from the owner of each participating dog. Owners and/or veterinarians were free to withdraw the dog from the study at any time. Owners were given a telephone number in case they had a question or observed an adverse effect. In compliance with GCP, clinicians either hold a French Certificate in Veterinary Dermatology or a Diploma of the European College of Veterinary Dermatology.

\subsection{Study Design}

The study was conducted as a double-blinded, randomized, controlled clinical trial in a teaching veterinary hospital. Each dog ear was considered as the experimental unit and randomization was based on the order of enrolment. The animals were observed twice at one week interval (intended period of study for each enrolled dog was $7 \pm 1$ day).

\subsection{Inclusion and Exclusion Criteria}

All dogs included were weaned (over 3 months old), client-owned, with clinical signs of otitis externa (including the presence of cerumen in the external ear canal), and without signs of marked and/or generalized skin disease at the time of inclusion (clinician assessment). Dogs with parasitic otitis, otic foreign body, end-stage proliferative ear disease, occlusive masses in the ear canal, ruptured tympanic membrane, autoimmune skin disease, or poor general health were not included in the trial. Dogs requiring a different treatment (product, procedure, frequency) and those showing an aggressive or fearful behaviour during the examination were also not included. 


\subsection{Prohibited Medications and Therapies}

Withdrawal times for prohibited medications were as follows: long-acting corticoids, 3 weeks; oral corticoids, 8 days; antihistamines, 3 days; oral antibacterial/antifungal agents, 8 days. All current treatments, including those eventually administered during the study, were noted in the concomitant table in compliance with the guidelines.

\subsection{Drug Administration}

\section{Ear cleaners tested}

Dogs were blindly randomized to receive cleaning with either Sonotix ${ }^{\circledast}$ (Vetoquinol, Lure France) or EpiOtic ${ }^{\circledR}$ Advanced (Virbac, Carros, France). The investigators were blinded to the type of ear cleaner received by dogs as the products were provided to investigators in identical bottles identified only by code numbers. The commercial ear cleaner EpiOtic ${ }^{\oplus}$ Advanced and the new formulation, Sonotix ${ }^{\oplus}$, were packaged in a commercial Good Manufacturing Practices (GMP) facility into $80 \mathrm{ml}$ polyethylene bottles with induction-nozzle caps. Bottles were labeled with the study reference, randomization number, batch number, and expiration date and owner notice.

In 8 dogs, ears cleaning were performed with Sonotix ${ }^{\oplus}$ containing ethoxydyglycol, capric glycerides, isopropryl alcohol, undecylenoyl and capryloyl glycine, glycerin, thromethamine, polysorbate 80 and calendula.

In the other 10 dogs, ears were cleaned with EpiOtic ${ }^{\oplus}$ Advanced containing salicylic acid, PCMX, EDTA, sodium docusate, the monosaccharides D-galactose, D-mannose and L-rhamnose, and nonionic surfactant excipients. Ears were cleaned by the veterinarians, the day of inclusion study, and daily by the owner by introducing the cleaning fluid directly into the ear canal and massaging the ear for 1 minute. The new formulation had been already used in a preliminary study on laboratory beagle dogs (data not shown) done to explore the harmlessness and tolerance of the formulation.

\section{OE treatment}

For the treatment of otitis, owners were instructed to administrate daily 10 drops of Aurizon ${ }^{\oplus}$ (Vetoquinol, Lure, France) 30 minutes after ear cleaning.

\subsection{Study Schedule and Variable Measures}

Following signed consent, baseline data (clinical history, concomitant medications, body weight, physical examination) ear cytology and video-otoscopy were recorded for each dog enrolled in the study. The effect of ear cleanser was assessed by the median of the scores of four video-otoscopic features (erythema, cerumen amount and thickness and the surface of ear canal covered by cerumen) and six cytological features (neutrophils, chromatin, yeast, cocci, rods and debris) Table 1 and Table 2 . Scorings were adjusted during a preliminary study on Beagle dogs (data not shown). Each ear was scored separately.

Otic exudate was collected by means of a cotton swab and the tip of the swab 
was then rolled onto a clean microscope slide. After drying, the slide was stained with a quick staining kit (RAL 555) and scored following a semi-quantitative method (range 0 - 4) with a total score of 0 to 20.

The erythema, the amount and thickness of cerumen and surface of external ear canal covered by ear wax were assessed by a video-otoscopic examination of each ear canal with individual scores. A four-point-scale and a five-point-scale were used to score the erythema and the cerumen, respectively, with a total score of 0 to 11 .

According to severity each video-otoscopic and cytological slides were evaluated blindly by one clinician. At the end of the study, each video-otoscopic and each cytological slide were blindly assessed by one clinician.

Both ears video-otoscopy and cytology were performed prior (T0) and 30 minutes ( $\mathrm{T} 0+30 \mathrm{~min})$ after the first ear cleaning at day 0 (D0), and during the follow-up visit scheduled on day 6 . Ear cleaning was performed by the clinician at D0 and subsequently done daily by the owner until day 5 (D5).

Table 1. Classification of the video-otoscopic scale of cerumen and erythema.

\begin{tabular}{|c|c|}
\hline Score & Description \\
\hline \multicolumn{2}{|c|}{ Cerumen amount and thickness } \\
\hline 0 & Cerumen virtually absent \\
\hline 1 & $\begin{array}{l}\text { Minor amount of thin plaques of cerumen. } \\
\text { No earwax plug. }\end{array}$ \\
\hline 2 & $\begin{array}{l}\text { Moderate amount of variable thickness and confluent plaques of } \\
\text { cerumen. No ear wax plug. }\end{array}$ \\
\hline 3 & $\begin{array}{l}\text { Great amount of thick and confluent plaques of cerumen. Presence of } \\
\text { small ear wax plug }\end{array}$ \\
\hline 4 & Massive and occlusive amounts of cerumen \\
\hline \multicolumn{2}{|c|}{ Surface of ear canal covered by cerumen } \\
\hline 0 & 0 \\
\hline 1 & $10 \%-30 \%$ \\
\hline 2 & $30 \%-50 \%$ \\
\hline 3 & $50 \%-75 \%$ \\
\hline 4 & $75 \%-100 \%$ \\
\hline \multicolumn{2}{|c|}{ Erythema } \\
\hline 0 & No erythema \\
\hline 1 & Very mild \\
\hline 2 & Mild \\
\hline 3 & Intense \\
\hline
\end{tabular}


Table 2. Classification of the microbiologic semi quantitative scale.

\begin{tabular}{|c|c|c|}
\hline Score & Organism & Count per oil field (x1000) \\
\hline 0 & & 0 \\
\hline 1 & & 1 \\
\hline 2 & Malassezia/Yeast & 2 \\
\hline 3 & & $2-5$ \\
\hline 4 & & $>5$ \\
\hline 0 & & 0 \\
\hline 1 & & $0-5$ \\
\hline 2 & Cocci & $5-15$ \\
\hline 3 & & $16-20$ \\
\hline 4 & & $>20$ \\
\hline 0 & & 0 \\
\hline 1 & & $0-5$ \\
\hline 2 & Rods & $5-15$ \\
\hline 3 & & $16-20$ \\
\hline 4 & & $>20$ \\
\hline 0 & & 0 \\
\hline 1 & & $0-5$ \\
\hline 2 & $\begin{array}{l}\text { Neutrophils and } \\
\text { chromatin ribbons }\end{array}$ & $5-15$ \\
\hline 3 & & $16-20$ \\
\hline 4 & & $>20$ \\
\hline 0 & & Clean background \\
\hline 1 & & Dirty background in one field \\
\hline 2 & $\begin{array}{c}\text { Dirty background } \\
\text { (debris, amorphous materials) }\end{array}$ & Dirty background in two fields \\
\hline 3 & & Dirty background in three fields \\
\hline 4 & & Dirty background in all fields \\
\hline
\end{tabular}

\subsection{Efficacy Outcome Measures}

Video-otoscopic and cytological scores were used to assess the primary efficacy in this study: baseline score (T0 on D0) and scoring at each time point (T0 + 30 min on D0 and D6).

Any abnormal clinical signs reported by the owner or identified by the investigator were recorded in the case report form.

\subsection{Data Analysis}

Dogs with one or major protocol deviations that affected the integrity of efficacy data were excluded from the analysis.

Qualitative parameters (sex, type of otitis, otitis history, number of adverse 
events) were compared using the fisher's exact or a Chi-test.

Animal characteristics were compared at baseline (D0) to check comparability before treatment. A non-parametric analysis was used due to the low sample number. Intra-group comparison of scores over the study period was performed using a Friedman analysis of variance (ANOVA), if significant, a Wilcoxon rank sum test was used for individual comparisons between time points. Inter-group comparisons of scores were analysed using a Mann Whitney Test.

A value of $\mathrm{p}<0.01$ was considered statistically significant. All analyses were performed using statistical R software (R Foundation for Statistical Computing, Vienna, Austria, 2014).

\section{Results}

\section{Population study}

A total of eighteen dogs comprising 8 males (four castrated) and 10 females (2 spayed), aged between 5 months old and 14 years old were enrolled in the study, and randomisation resulted in 10 dogs in EpiOtic ${ }^{\circledast}$ Advanced and 8 dogs in Sonotix ${ }^{\oplus}$. Breeds represented were 6 Cocker spaniels (3 from one breeder), 4 Labradors, 2 Golden Retrievers, 2 French Bulldogs, 1 Poodle and 3 cross breed dogs. The dogs weighed between $2.6 \mathrm{~kg}$ and $42.6 \mathrm{~kg}$.

The descriptive characteristics of the dogs, the medical history and the scores were not significantly different on Day 0 . Pendulous ears were overall overrepresented but no significant differences were observed between groups. The sex ratio 1:1 was balanced and the majority of dogs had bilateral otitis in both groups (80\% EpiOtic ${ }^{\circledast}$ Advanced, 87\% Sonotix ${ }^{\circledast}$ ). Fifteen dogs had bilateral and 3 had unilateral otitis externa on presentation, giving a total of 33 affected ears; 8 dogs presented as new cases of otitis, whereas the remaining 10 were undergoing recrudescence of a prior episode (no significant difference among groups).

Cytological and video-otoscopic clinical examination

Cytological samples of the otic exudate at T0 revealed pure Malassezia yeast overgrowth in 17 of 33 ears (51.5\%), rods and cocci in 8 of 33 ears (24.2\%), cocci and Malassezia in 6 of 33 ears (18.2\%) and rods, cocci and Malassezia in 2 of 33 ears (6.1\%). Median of global cytological scores at T0 was 6 for Sonotix ${ }^{\circledast}$ and 5 for EpiOtic ${ }^{\circledast}$ Advanced (Table 3 ). The important reductions in yeast, cocci, and rod-shaped organism counts were demonstrated in smears 30 minutes after the cleansing in both groups and at D6 (Figure 1).

Table 3. Median global scores of the video-otoscopic and cytological examinations for EpiOtic ${ }^{\circledast}$ Advanced and Sonotix ${ }^{\otimes}{ }^{a}$ significantly different from baseline value $(\mathrm{p}<0.01)$.

\begin{tabular}{ccccccc}
\hline & \multicolumn{3}{c}{ Cytological global median score } & \multicolumn{2}{c}{ Video-otoscopic global median scores } \\
\hline & T0 & T0 +30 min & D6 & T0 & T0 + 30 min & D6 \\
\hline $\begin{array}{c}\text { Sonotix } \\
\text { EpiOtic } \\
\text { Advanced }\end{array}$ & 5 & $2^{\mathrm{a}}$ & $2^{\mathrm{a}}$ & 6 & $3^{\mathrm{a}}$ & $2^{\mathrm{a}}$ \\
\hline
\end{tabular}




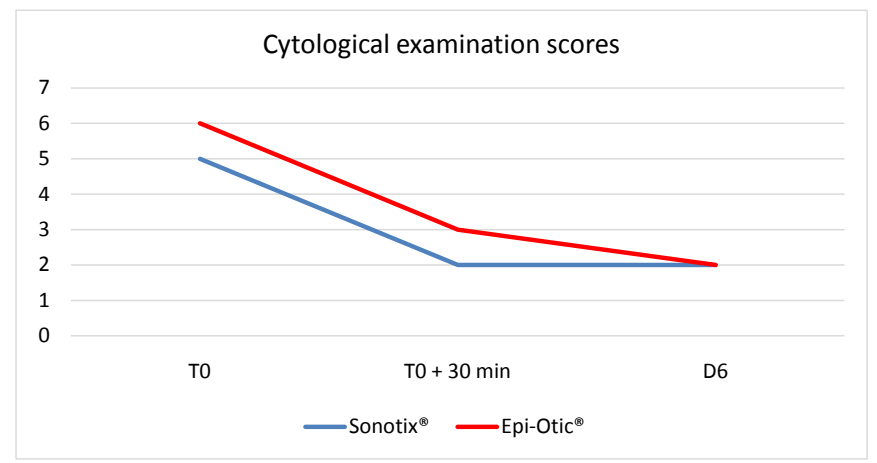

Figure 1. Median global scores for EpiOtic ${ }^{\circledast}$ Advanced and Sonotix ${ }^{\circledast}$ before and after treatment.

Intra-group comparison shows a significant difference ( $\mathrm{p}$ value $<0.01$ ) between the median of global cytological scores between T0 (Sonotix ${ }^{\oplus}: 5$, EpiOtic ${ }^{\circ}$ Advanced: 6) and T0 + 30 min (Sonotix ${ }^{\circledR}: 2$, EpiOtic ${ }^{\circledR}$ Advanced: 3 ) and T0 and D6 (Sonotix ${ }^{\oplus}: 2$, EpiOtic ${ }^{\oplus}$ Advanced: 2). Inter-group comparison shows no statistical difference at $\mathrm{T} 0+30 \mathrm{~min}$ and $\mathrm{D} 6(\mathrm{P}<0.01)$.

Almost all ears (26 ears - 78.8\%) had at T0 some degree of erythema on initial presentation, and erythema was rated as very mild in 17 ears (51.5\%), mild in 7 ears (21.2\%) and intense in 2 ears (6.1\%).

All ears had a variable amount of cerumen: 18 (54.5\%) external ear canals were entirely covered by confluent plaques of cerumen, 9 (27.3\%) were partially covered by confluent plaques of cerumen, 5 (15.2\%) were patchily covered by non-confluent plaques of cerumen and $1(3 \%)$ was completely occluded by a massive amount of cerumen.

The median global scores of video-otoscopic examination, as shown in Table 3 and Figure 2, was 6 at T0 for both groups, 3 for Sonotix ${ }^{\oplus}$ and 4 for EpiOtic ${ }^{\star}$ Advanced at $\mathrm{T} 0+30 \mathrm{~min}$ and 2 at $\mathrm{D} 6$ for both group.

30 minutes after the cleaning of ears there was a significant $(\mathrm{P}<0.01)$ decrease of the amount of cerumen in vertical and horizontal ear canal from baseline. Intra-group comparison shows a significant difference ( $\mathrm{p}$ value $<0.01$ ) between T0 (Sonotix ${ }^{\oplus}: 6$ EpiOtic $^{\circledast}$ Advanced: 6 ) and T0 +30 min (Sonotix ${ }^{\oplus}: 3$, EpiOtic ${ }^{\star}$ Advanced: 4) and between T0 and D6 (Sonotix ${ }^{\oplus}: 2$, EpiOtic ${ }^{\oplus}$ Advanced: 2).

Inter-group comparison shows a significant difference at $\mathrm{T} 0+30 \mathrm{~min}(\mathrm{p}<$ 0.01 ) between Sonotix ${ }^{\oplus}$ (median score: 3 ) and EpiOtic ${ }^{\oplus}$ Advanced (median score: 4) (Figure 3).

\section{Discussion}

In the present study, both ear cleansers reduced bacterial and yeast overgrowth and clinical signs (cerumen burden, erythema) in a population of client-owned dogs with spontaneous OE. The cleansers decreased ear wax, as early as $30 \mathrm{~min}$ utes, as evidenced by marked reduction of amount and thickness of cerumen and surface of external ear canal covered by ear wax. 


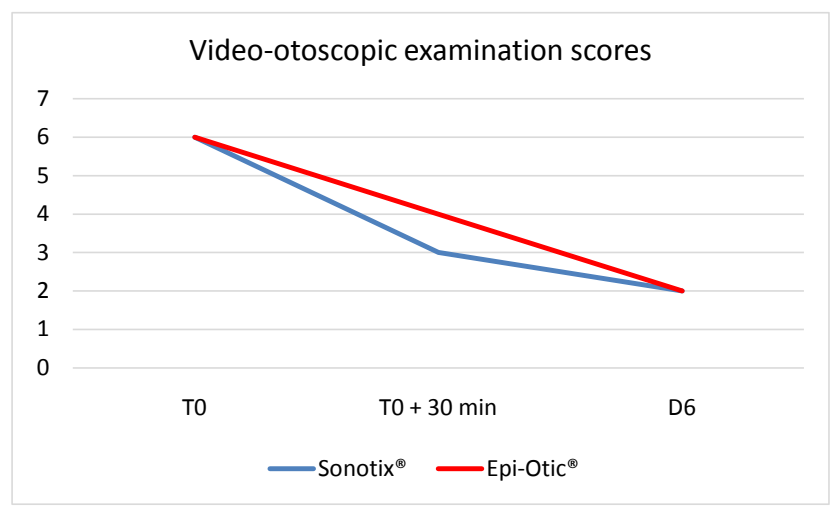

Figure 2. Median global scores for EpiOtic ${ }^{\circledast}$ Advanced and Sonotix ${ }^{\circledast}$ before and after treatment.

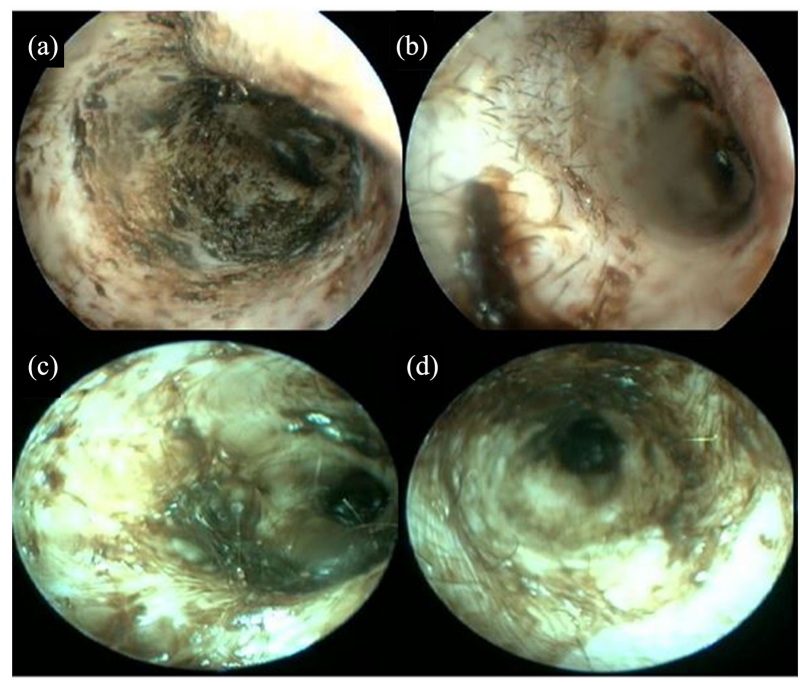

Figure 3. Video-otoscopy images showing the external auditory canals of dogs before and 30 minutes after receiving ear cleaning with either Sonotix ${ }^{\oplus}$ or Epi-Otic ${ }^{\oplus}$ Advanced. (a) External ear canal of a dog before receiving Sonotix ${ }^{\circledast}$ (T0) (b) The same external ear canal as showed in image a, 30 minutes after cleaning with Sonotix ${ }^{\otimes}(\mathrm{T} 0+30 \mathrm{~min})(\mathrm{c})$ External ear canal of a dog before receiving Epi-Otic ${ }^{\circledast}$ Advanced (T0) (d) The same external ear canal as showed in image c, 30 minutes after cleaning with Epi-Otic ${ }^{\circledast}$ Advanced (T0 $+30 \mathrm{~min}$ ).

However, the cleaning activity of Sonotix ${ }^{\oplus}$ was statistically superior to Epi-Otic ${ }^{\circledast}$ Advanced as evidenced by the median global scores of video-otoscopic examination at T $30 \mathrm{~min}(\mathrm{p}<0.01)$.

This suggests that Sonotix ${ }^{\otimes}$ has a faster and more efficient action in cerumen removal. This enhanced ceruminolytic activity had also been demonstrated in an in vitro trial [17]. In that study, which the aim was to compare the ceruminolytic activity of five commercial ear cleaners on synthetic canine cerumen, the ear solution (Sonotix ${ }^{\oplus}$, Vetoquinol, Lure, France) had the most effective and rapid ceruminolytic activity of the products tested. This fastest ceruminolytic activity could be in part explained by ingredient composition comprising three cerumenolytic agents acting synergistically. Ethoxydiglycol (protic solvent) and iso- 
propyl alcohol (organic solvent) disrupt the integrity of earwax by lysing the agglomerate and by breaking the bond between corneocytes whereas capric glycerides expedite the ceruminolytic and cleaning process by emulsifying debris. Cytological and clinical improvement, compared to T0, was also seen at D6 but no difference was seen between Sonotix ${ }^{\oplus}$ and EpiOtic ${ }^{\oplus}$ Advanced. As these dogs had clinical OE, for ethical reasons they were not treated with ear cleaner only. Both groups were prescribed daily application of Aurizon ${ }^{\oplus}$, a product known to have strong anti-inflammatory, antibacterial and anti-yeast activity.

The video-otoscopic scoring used in this study was developed to assess the cerumen burden and erythema as opposed to other published scorings developed to globally assess otitis externa severity [21]. In order to reduce inter clinician bias and before and after application bias, all video-otoscopy and cytology were evaluated by an experienced dermatologist (Dip. ECVD).

In healthy ear, the removal of cerumen and debris from the external ear canal is primarily achieved by epithelial migration. This physiologic process allows cerumen transport away from the tympanic membrane and towards the opening at the distal end of the ear canal. Recently this process has been demonstrated to follow both radial and centrifugal patterns in the dog [22]. While it is commonly accepted ear cleaning is not necessary for healthy ears, it has several benefits in many conditions as those in which epithelial migration is impaired. Failure of epithelial migration may result in the accumulation of flakes of skin in the ear canal and soft wax plugs. Furthermore, the migratory process is a key factor in the repair of spontaneous TM perforations and post-operative TM incisions

Effective ear cleaning is an essential part of any treatment scheme because it favours the contact between the ointment and the lining ear epidermis and speeds resolution by the removal of cerumen, microbial organisms, cellular debris and free fatty acids and prevents potential inactivation of topical therapy by purulent material [4] [13]. Our results comfort the common habit of prescribing a regular ear cleaner as a component in the management of $\mathrm{OE}$ in dogs.

A wide range of ear cleansing preparations and procedures aimed to remove exudates and ceruminous debris have become very popular in veterinary practice. Knowledge of their properties helps to choose the best available product for specific clinical situations. Choosing the most appropriate product is akin to selecting a shampoo. It depends on the individual patient and it is of crucial importance to fully evaluate the primary, predisposing and perpetuating factors, the type otitis and the chosen topical therapy.

Oil-based ear cleaners, compared to those containing strong ceruminolytics, tend to be much gentler as they loosen wax, rather than disrupt it, but they do leave a residue in the canal. Moreover, it is questionable if they are of many benefits in ears with a purulent discharge. A cleaner with aqueous properties would be more indicated in a purulent otitis [23].

Knowledge of ear cleaner's pH it is also very important, especially if they are associated with topical antibiotics. Even if the antimicrobial activity of certain 
ear cleaners has been shown to be associated with a low $\mathrm{pH}$, acid cleaners may inactive some antimicrobials (especially aminoglycosides and fluoroquinolones) [24]. In the present study, a complete clinical and microbiological resolution was observed at D6 suggesting that the concomitant use of Sonotix ${ }^{\circledR}$, didn’t interfere with the activity of Aurizon ${ }^{\circledast}$ or didn't inactivate the antimicrobial contained in it. Further studies to directly prove it (synergy in vitro test) are however necessary.

\section{Funding Statement}

This study was sponsored by Vetoquinol Lure, France

\section{Conflicts of Interest}

J. F. Jamet: None Declared; G. Marignac: None Declared; J. Y. Petit: None Declared; J. L. Petit, F. Woehrlé, T. Trouchon and O. Fantini are employees of Vetoquinol; S. Perrot: None Declared.

\section{References}

[1] Carlotti, D.N. (1991) Diagnosis and Medical Treatment of Otitis Externa in Dogs and Cats. Journal of Small Animal Practice, 32, 394-400.

https://doi.org/10.1111/j.1748-5827.1991.tb00963.x

[2] Huang, H.-P., Little, C.J.L. and McNeil, P.E. (2009) Histological Changes in the External Ear Canal of Dogs with Otitis Externa. Veterinary Dermatology, 20, 422-428. https://doi.org/10.1111/j.1365-3164.2009.00853.x

[3] Radlinsky, M.G. and Mason, D.E. (2005) Diseases of the Ear. Textbook of Veterinary Internal Medicine, 2, 6.

[4] McKeever, P.J. and Torres, S.M.F. (1997) Ear Disease and Its Management. Veterinary Clinics of North America: Small Animal Practice, 27, 1523-1536. https://doi.org/10.1016/S0195-5616(97)50137-9

[5] Gotthelf, L.N. (2005) Factors That Predispose the Ear to Otitis Externa. In: Small Animal Ear Diseases (An Illustrated Guide), 2nd Edition, Elsevier, Amsterdam, 141-171. https://doi.org/10.1016/B0-72-160137-5/50010-6

[6] Angus, J.C., Lichtensteiger, C., Campbell, K.L. and Schaeffer, D.J. (2002) Breed Variations in Histopathologic Features of Chronic Severe Otitis Externa in Dogs: 80 Cases (1995-2001). Journal of the American Veterinary Medical Association, 221, 1000-1006. https://doi.org/10.2460/javma.2002.221.1000

[7] Crespo, M.J., Abarca, M.L. and Cabanes, F.J. (2002) Occurrence of Malassezia Spp. in the External Ear Canals of Dogs and Cats with and without Otitis Externa. Medical Mycology, 40, 115-121.

[8] Foster, A.P. and DeBoer, D.J. (1998) The Role of Pseudomonas in Canine Ear Disease. Compendium of Continuing Education for the Practicing Veterinarian, 20, 909-919.

[9] Junco, M.T. and Barrasa, J.L. (2002) Identification and Antimicrobial Susceptibility of Coagulase Positive Staphylococci Isolated from Healthy Dogs and Dogs Suffering from Otitis Externa. Journal of Veterinary Medicine Series B, 49, 419-423. https://doi.org/10.1046/j.1439-0450.2002.00571.x

[10] Morris, D.O. (1999) Malassezia Dermatitis and Otitis. Veterinary Clinics of North 
America: Small Animal Practice, 29, 1303-1310. https://doi.org/10.1016/S0195-5616(99)50128-9

[11] Ginel, P.J., Lucena, R., Rodriguez, J.C. and Ortega, J. (2002) A Semiquantitative Cytological Evaluation of Normal and Pathological Samples from the External Ear Canal of Dogs and Cats. Veterinary Dermatology, 13, 151-156. https://doi.org/10.1046/j.1365-3164.2002.00288.x

[12] Tater, K.C., Scott, D.W., Miller, W.H. and Erb, H.N. (2003) The Cytology of the External Ear Canal in the Normal Dog and Cat. Journal of Veterinary Medicine Series A, 50, 370-374. https://doi.org/10.1046/j.1439-0442.2003.00548.x

[13] Nuttall, T. and Cole, L.K. (2004) Ear Cleaning: The UK and US Perspective. Veterinary Dermatology, 15, 127-136. https://doi.org/10.1111/j.1365-3164.2004.00375.x

[14] Lloyd, D.H., Bond, R. and Lamport, I. (1998) Antimicrobial Activity in Vitro and in Vivo of a Canine Ear Cleanser. Veterinary Record, 143, 111-112. https://doi.org/10.1136/vr.143.4.111

[15] Swinney, A., Fazakerley, J., McEwan, N. and Nuttall, T. (2008) Comparative in Vitro Antimicrobial Efficacy of Commercial Ear Cleaners. Veterinary Dermatology, 19, 373-379. https://doi.org/10.1111/j.1365-3164.2008.00713.x

[16] Rème, C.A., et al. (2006) The Efficacy of an Antiseptic and Microbial Anti-Adhesive Ear Cleanser in Dogs with Otitis Externa. Veterinary Therapeutics, 7, 15-26.

[17] Brevet, A., Moreau, M. and Petit, J.L. (2015) Comparative in Vitro Ceruminolytic Activity of 5 Commercialized Veterinary Ear Cleaners and a New Ear Cleaner Prototype. 21 st FECA VA Eurocongress, Barcelona, Spain, 15-17 October 2015.

[18] Mansfield, P.D., Steiss, J.E., Boosinger, T.R. and Marshall, A.E. (1996) The Effects of Four, Commercial Ceruminolytic Agents on the Middle Ear. Journal of the American Animal Hospital Association, 33, 479-486. https://doi.org/10.5326/15473317-33-6-479

[19] Nielloud, F., et al. (2004) Development of an in Vitro Test to Evaluate Cerumen Dissolving Properties of Several Veterinary Ear Cleansing Solutions. Journal of Drug Delivery Science and Technology, 14, 235-238. https://doi.org/10.1016/S1773-2247(04)50106-5

[20] Sánchez-Leal, J., Mayos, I., Homedes, J. and Ferrer, L. (2006) In Vitro Investigation of Ceruminolytic Activity of Various Otic Cleansers for Veterinary Use. Veterinary Dermatology, 17, 121-127. https://doi.org/10.1111/j.1365-3164.2006.00504.x

[21] Nuttall, T. and Bensignor, E. (2014) A Pilot Study to Develop an Objective Clinical Score for Canine Otitis Externa. Veterinary Dermatology, 25, 530-537. https://doi.org/10.1111/vde.12163

[22] Tabacca, N.E., Cole, L.K., Hillier, A. and Rajala-Schultz, P.J. (2011) Epithelial Migration on the Canine Tympanic Membrane. Veterinary Dermatology, 22, 502-510. https://doi.org/10.1111/j.1365-3164.2011.00982.x

[23] Forsythe, P.J. (2016) Acute Otitis Externa: The Successful First-Opinion Ear Consultation. In Practice, 38, 2-6. https://doi.org/10.1136/inp.i412

[24] Nuttal, T. (2016) Successful Management of Otitis Externa. In Practice, 38, 17-21. https://doi.org/10.1136/inp.i1951 